\title{
How Well Do We Know Atomic Motions of Simple Liquids?
}

\author{
C. Cabrillo, ${ }^{1,2}$ F. J. Bermejo, ${ }^{1,2}$ M. Alvarez, ${ }^{2, *}$ P. Verkerk, ${ }^{3, \dagger}$ A. Maira-Vidal, ${ }^{2}$ S. M. Bennington, ${ }^{4}$ and D. Martín ${ }^{4}$ \\ ${ }^{1}$ Department of Electricity and Electronics, University Basque Country, P.O. Box 644, E-48080 Bilbao, Spain \\ ${ }^{2}$ Consejo Superior de Investigaciones Científicas, Serrano 119-123, E-28006 Madrid, Spain \\ ${ }^{3}$ Interfacultair Reactor Instituut, Technische Universiteit Delft, $2629 \mathrm{JB}$ Delft, The Netherlands \\ ${ }^{4}$ Rutherford Appleton Laboratory, Chilton, Didcot, Oxon Ox11 OQX, United Kingdom
}

(Received 25 March 2002; published 30 July 2002)

\begin{abstract}
Microscopic motions in molten potassium spanning three frequency decades are studied by neutronscattering techniques. These comprise well-defined density oscillations and stochastic particle rearrangements and both are modeled on microscopic grounds. While vibratory motions are shown to share characteristics with those of their parent crystals, dynamic correlations between a diffusing particle and its neighbors can be accounted for only semiquantitatively.
\end{abstract}

DOI: 10.1103/PhysRevLett.89.075508

PACS numbers: 61.25.Mv, 61.12.-q, 62.30.+d

Our view of the microscopic dynamics of monoatomic liquids heavily relies upon findings on molten alkali metals that are paradigms of the so called "simple (harmonic)liquid" behavior [1]. Atomic motions are there rationalized in terms of particle configurations. Those vibrational in nature take place whenever atoms within a configuration oscillate for times longer than their characteristic periods, leading to spectra showing well-defined peaks up to large $Q$ wave vectors. In contrast, stochastic motions such as those involving mass diffusion can be thought of as taking place once the configurations (or "cages") become unstable leading to local rearrangements which restore local equilibrium [2]. The quantity amenable to experiment is the $S(Q, \omega)$ dynamic structure factor. Its intensity often shows, in addition to well-defined excitation peaks [3], zero-frequency (quasielastic) components due to spacedependent flow motions. These are known to be highly entangled since the diffusing particle can move to a new position only if it has been left vacant before, and therefore a significant correlation between the moving particle and its surroundings surely exists. Such intricacies force the analysis of experimental $S(Q, \omega)$ [3] to rely upon a number of simplifying assumptions. Its inelastic component $S^{\text {inel }}(Q, \omega)$ containing details about collective density fluctuations is often modeled by response functions such as the damped-harmonic oscillator (DHO), valid within the hydrodynamic realm or interpolation formulas connecting hydrodynamic and microscopic regimes [4]. Also, results from kinetic theory [5] have proven adequate for analysis of data concerning hard sphere fluids. However, to account for the observed spectral line shape of a dense liquid metal these models often have to be supplemented by ad hoc quasielastic components which may introduce some model dependence in the derived physical quantities.

Here we report on the dynamics of molten $\mathrm{K}$ studied by a combination of neutron-scattering techniques at $T=$ 343 K. Previous neutron data measured over a restricted portion of the $Q, \omega$ plane [6] portray the dynamics close to melting $\left(T_{m}=336.7 \mathrm{~K}\right)$ as markedly different from other alkali metals. Diffusive motions as seen in $S_{i}^{\text {q.el }}(Q, \omega)$, the incoherent part of the quasielastic spectra, are found to be "solidlike" [6], having large residence times for a particle within a "cage." Naturally abundant K is a mostly coherent scatterer of neutrons (i.e., coherent to incoherent cross sections $\left.\sigma_{c} / \sigma_{i}=6.26\right)$. This allows the derivation of the coherent dynamic structure factor from the measured cross section while enabling a separate study of $S_{i}^{\text {q.el }}(Q, \omega)$, that is the dominant feature at low $Q$ 's.

Two sets of measurements were carried out at the ISIS source (U.K.) on a sample contained within a custom-built furnace. The IRIS backscattering spectrometer was set up using the [002] reflection of pyrolitic graphite for the analyzer crystals providing an energy resolution $\Delta E \approx$ $2.4 \mathrm{GHz}$. The MARI chopper spectrometer was employed using incident energies $E_{i}=3.6 \mathrm{THz}(\Delta E \approx 0.1 \mathrm{THz})$, $7.2 \mathrm{THz}(\Delta E \approx 0.24 \mathrm{THz})$, and $12 \mathrm{THz}(\Delta E \approx$ $0.3 \mathrm{THz}$ ). Measurements on IRIS and those on MARI at low $E_{i}$ were devoted to a proper modeling of $S^{\text {q.el }}(Q, \omega)$ including both coherent and incoherent components. Measurements at MARI with higher $E_{i}$ 's aimed to the precise determination of $S^{\text {inel }}(Q, \omega)$. The measured intensity $I(Q, \omega)$ after correcting for self-attenuation, multiplescattering, and multiexcitation effects fulfills

$$
\begin{aligned}
& I(Q, \omega)=A[S(Q, \omega) \otimes R(Q, \omega)] \\
& S(Q, \omega)=\frac{\sigma_{i}}{\sigma_{i}+\sigma_{c}} S_{i}(Q, \omega)+\frac{\sigma_{c}}{\sigma_{i}+\sigma_{c}} S_{c}(Q, \omega) .
\end{aligned}
$$

Here $A$ is a global scaling constant, $R(Q, \omega)$ is the instrument resolution function, and $S_{i}(Q, \omega)$ and $S_{c}(Q, \omega)$ are, respectively, the incoherent and coherent-scattering components weighed by their relative cross sections.

Let us first deal with quasielastic spectra $S^{\text {q.el }}(Q, \omega)$. Its incoherent part $S_{i}^{\text {q.el }}(Q, \omega)$ is the dominant contribution for $Q$ below $Q_{p} \approx 1.6 \AA^{-1}$, where $S(Q)$ shows its main maximum. Its linewidth is easily determined on IRIS after allowance of a second, weaker and broader component arising from the coherent part $S_{c}^{\mathrm{q} . \mathrm{el}}(Q, \omega)$. The line shape 
of $S^{\text {q.el }}(Q, \omega)$ is well accounted for using two Lorentzians with adjustable widths. Accurate measurement of $S_{c}^{\mathrm{q} . \mathrm{el}}(Q, \omega)$ requires a wider energy window as provided by measurements on MARI carried out with $E_{i}=$ 3.6 THz. Within $1.6 \AA^{-1} \leq Q \leq 1.8 \AA^{-1}$ the separation of both contributions is again facilitated by a large difference in linewidth. For $1.1 \AA^{-1} \leq Q \leq 1.3 \AA^{-1}$ both contributions show comparable linewidths and the separation is done relying on the mode-coupling prescription $[3,7]$. It predicts a quasielastic spectrum with linewidth $\Delta \omega(Q)$ and amplitude $S_{i}^{\text {q.el }}(Q, 0)$,

$$
\begin{gathered}
\Delta \omega(Q)=D Q^{2}-H(\delta) Q / Q^{*}, \\
S_{i}^{\text {q.el }}(Q, 0)=\left[1+G\left(\delta^{-1}\right) Q / Q^{*}\right] / \pi D Q^{2} .
\end{gathered}
$$

Here $D$ is the self-diffusion coefficient, $Q^{*}=$ $16 \pi M n D^{2} \beta, M$ is the particle mass, $n$ is the number density, $\delta=D /(D+\eta / M n)$ with $\eta$ being the shear viscosity [8], and $\beta=1 / k_{B} T$. The functions $G\left(\delta^{-1}\right)$ and $H(\delta)$ are given in Ref. [3]. The first terms in Eq. (2) stand for Fickian diffusion and the second account for the coupling of mass diffusion to the collective modes. The adequacy of Eq. (2) is demonstrated in Fig. 1 which compares the wave vector dependence of the linewidth as determined on IRIS with $\Delta \omega(Q)$ within the two $Q$ ranges where it could be safely separated. On such a basis we choose Eq. (2) to represent $S_{i}^{\text {q.el }}(Q, \omega)$ without involving any free parameter and then make a reestimation of the coherent linewidth. Its $Q$ dependence, also plotted in Fig. 1, shows a minimum about $Q_{p}$ and can be semiquantitatively repro-

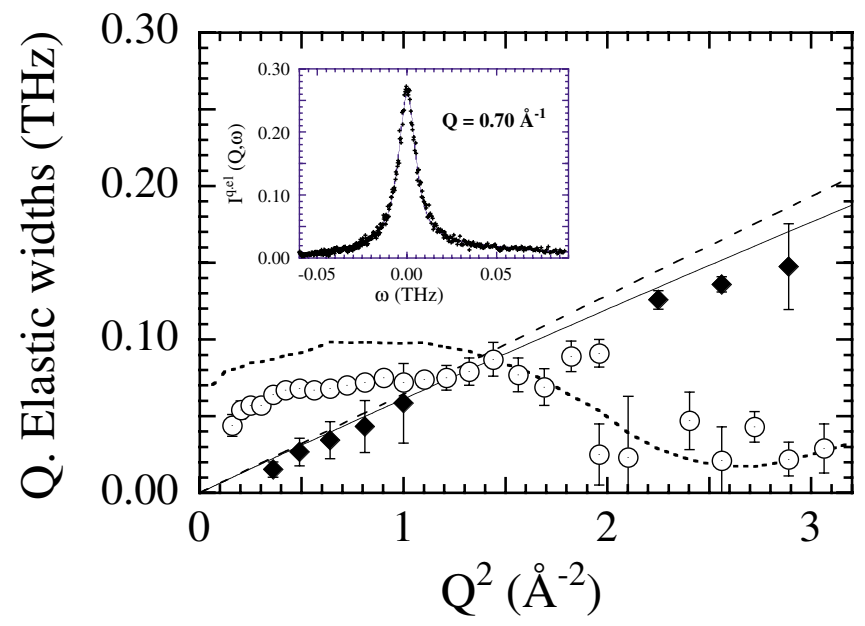

FIG. 1 (color online). $\quad Q$ dependence of quasielastic linewidths. The solid line depicts the prediction of Eq. (2) and dashes indicate the Fickian $\Delta \omega=D Q^{2}$ linewidths. Solid lozenges show the linewidth for $S_{i}^{\mathrm{q} . \mathrm{el}}(Q, \omega)$ as determined on IRIS. The open circles with a dot show the coherent quasielastic widths for $S_{c}^{\mathrm{q} . \mathrm{el}}(Q, \omega)$ as determined from MARI. The dotted line shows the prediction of Eq. (4). The inset depicts spectra for a representative $Q=0.70 \AA^{-1}$ value. duced from [9]

$$
\Delta \omega_{c}(Q)=\frac{\Delta \omega(Q)}{S(Q)\left[1-j_{0}\left(Q R_{0}\right)+2 j_{2}\left(Q R_{0}\right)\right]} .
$$

Here $R_{0}$ is an atomic diameter set to the value corresponding to the main minimum in the $V(r)$ pair interaction potential [10], $j_{x}()$ stand for spherical Bessel functions, and use is made of $\Delta \omega(Q)$ instead of Fickian diffusion. The picture behind this portrays quasielastic scattering as arising from diffusive motions that enable a density fluctuation to relax. Their relevant time scale is within $\approx 1-10 \mathrm{ps}$. Because of the relatively high fluid density, single-particle diffusion will be slower than that expected for hydrodynamic diffusion due to coupling with its surrounding particles. Such coupling gives rise to the modulation of $\Delta \omega_{c}(Q)$ with $Q$, while its amplitude follows the oscillations of $S(Q)$.

To analyze $S^{\text {inel }}(Q, \omega)$ we seek an alternative representation for $S_{c}^{\text {inel }}(Q, \omega)$ that extends the usual "three-pole approximation" [4]. This is needed since the standard formulation [4] predicts a spectrum consisting of two inelastic peaks plus a small quasielastic peak with a linewidth $\propto Q$ that significantly departs from the data shown in Fig. 1. To improve the representation of $S_{c}^{\text {inel }}(Q, \omega)$ we write

$$
S_{c}^{\text {inel }}(Q, \omega)=\frac{(\omega \beta) S(Q)}{1-e^{(-\hbar \omega \beta)}} \operatorname{Re}[i \omega+\tilde{M}(Q, i \omega)]^{-1},
$$

where the tilde stands for Laplace transform. The memory function is specified by a continued fraction $\tilde{M}(Q, s)=$ $\omega_{0}^{2}\left[s+\tilde{K}^{(1)}(Q, s)\right]^{-1} \quad$ with $\quad \tilde{K}^{(n)}(Q, s)=K^{(n)}(Q, t=$ $0)\left[s+\tilde{K}^{(n+1)}(Q, s)\right]^{-1}$. The usual approach terminates the continued fraction at $n=1$ assuming that $K^{(2)}(Q, t)$ decays too fast within our time window; i.e., $\tilde{K}^{(2)}=1 / \tau$. This is improved by going one step further. In doing so one arrives at

$$
\tilde{M}(Q, s)=\omega_{0}^{2}\left[s+\frac{\omega_{l}^{2}-\omega_{0}^{2}}{s+\frac{\left(\omega_{s}^{4}-\omega_{l}^{4}\right)}{\left(\omega_{l}^{2}-\omega_{0}^{2}\right)(s+1 / \tau)}}\right]^{-1},
$$

where $\omega_{0}^{2}$ is the second frequency moment normalized to $S(Q)$ while $\omega_{l}^{2}$ and $\omega_{s}^{4}$ are, respectively, the ratios between the fourth and sixth moments and $\omega_{0}^{2}$. The second moment is calculated from $\omega_{0}^{2}=Q^{2} / \beta M S(Q)$ with the data taken from Ref. [11], while the fourth and the sixth depend upon details of the interaction potential. Direct determination of $\omega_{l}$ from experiment is precluded by background noise as well as by limitations imposed by the neutron kinematics, and therefore use is made of an approximation giving $\omega_{l}$ in terms of $R_{0}$ and an "Einstein frequency" $\omega_{E}$ [4].

Figure 2 compares spectra to the model given by Eq. (5) using $\tau, \omega_{s}, \omega_{l}$ and the scale factor as adjustable parameters. The initial estimates for $R_{0}=4.72 \AA$ and $\omega_{E}=$ $1.2 \mathrm{THz}$ correspond to the minimum of $V(r)$ and to $\omega_{E}=$ $\left[4 \pi \rho / 3 M \int_{0}^{\infty} d r r^{2} g(r) V^{\prime \prime}(r)\right]^{1 / 2} \quad[4,10]$, where primes 
denote the second derivative and $g(r)$ denotes the radial distribution function. They were refined iteratively using the whole set of spectra and the final values were $R_{0}=$ $4.62 \AA, \omega_{E}=1.55 \mathrm{THz}$. In contrast, unphysically large values for $\omega_{E}$ result if the standard expression for $S_{c}^{\text {inel }}(Q, \omega)[4]$ is used to fit the spectrum.

Clear inelastic peaks arising from propagating density oscillations are seen for $Q \leq 1.3 \AA$. Such a $Q$ value marks an abrupt change in the propagation regime as shown by the characteristic time $\tau$ displayed in Fig. 2. It also sets the time scale for vibratory motions which, for $0.5 \AA^{-1} \leq$ $Q \leq 1 \AA^{-1}$, comes out to be 1 order of magnitude shorter

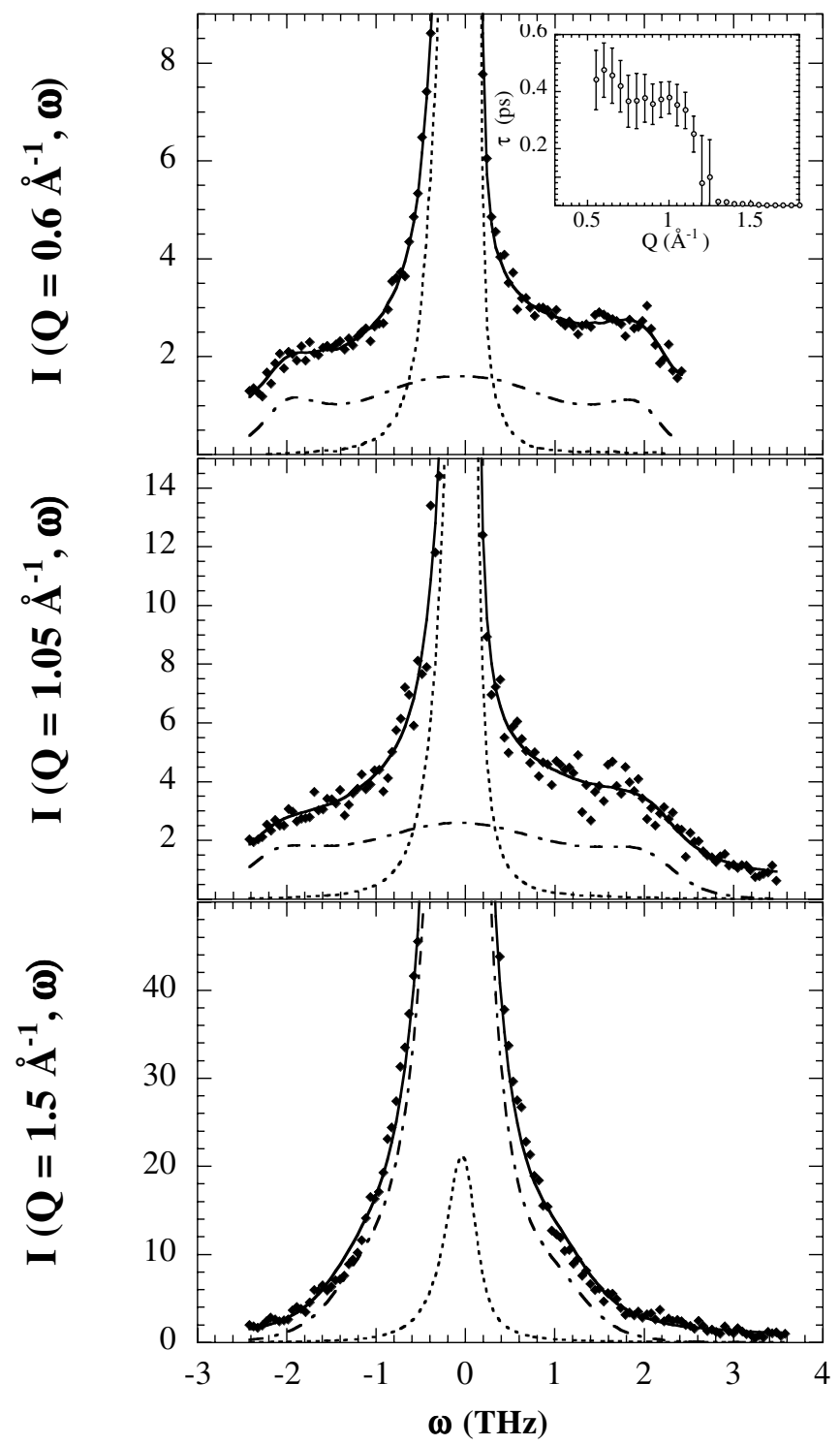

FIG. 2. Spectra covering large energy transfers. Symbols depict experimental intensities while thick solid lines show the fitted model using Eq. (5). The dashed line shows the symmetrized inelastic intensity as predicted by Eq. (5) and the dotted line depicts the quasielastic contribution. The inset shows the characteristic time $\tau$. than those of stochastic nature. Plots for $\omega_{0}$ and $\omega_{l}$ together with results for the latter if left as a free parameter are shown in Fig. 3(a). The closeness between calculated and fitted values for $\omega_{l}$ validates the use of Eq. (5) and suggests that the reduced fourth-frequency moment should not be too far from our approximation.

The linear dispersion $c_{T} Q$ given by the isothermal sound velocity $c_{T}=1605 \mathrm{~ms}^{-1}$ approaches $\omega_{0}$ for $Q \leq$ $0.3 \AA^{-1}$. The frequencies $\omega_{m}$ corresponding to maxima of peaks in $S(Q, \omega)$ and those $\omega_{m l}$ from maxima of the longitudinal current $C_{L}(Q, \omega)=\omega^{2} S(Q, \omega) / Q^{2}$ calculated from the fitted spectra are significantly above $\omega_{0}$. Since $\omega_{m l}$ are equivalent to the characteristic frequencies of a DHO they may be considered as true physical frequencies of the oscillatory motion. Damping effects become increasingly important for $Q>0.6 \AA^{-1}$. The difference between $\omega_{m l}$ and $\omega_{m}$ provides a way of quantifying its importance. Moreover, for $Q>1.3 \AA^{-1}$, the absence of any clear peak in $S(Q, \omega)$ constitutes a vivid reminder that excitations are here overdamped. For $Q>$ $1.3 \AA^{-1}, \omega_{m l}$ should then be regarded as a statistical tool to describe the data rather than as a physical frequency.

Both $\omega_{m}, \omega_{m l}$ and $\omega_{l}$ approach the $Q \rightarrow 0$ hydrodynamic realm in a way reminiscent of that followed by other alkali metals [3] showing a large amount of positive anomalous dispersion. Our data approach a linear dispersion given by the high-frequency sound velocity $c_{\infty}=$ $\sqrt{(3 / \beta M)+\left(3 \omega_{E}^{2} R_{0}^{2} / 10\right)}=2490 \mathrm{~ms}^{-1}$ of an elastic medium being sampled "instantaneously" by a highfrequency probe. The mechanisms leading to such large velocity dispersion are understood from the breakdown of the linearized Navier-Stokes formulas to describe hydrodynamic phenomena for frequencies above $\mathcal{B}_{\mathrm{T}} / \eta \approx 0.6 \mathrm{THz}$, where $\mathcal{B}_{\mathrm{T}}$ is the isothermal bulk modulus [13]. Here, the propagation of dilatational or shear waves is determined from the equations of motion for an isotropic solid in which the bulk and rigidity moduli are now complex, frequency dependent quantities. A connection with observed quantities is made in terms of the $Q$-dependent modulus [4]: $C_{11}(Q)=\frac{M n \omega_{l}^{2}}{Q^{2}}$, which enables one to get information on $C_{11}(Q)$ from knowledge of $\omega_{l}$. Figure 3(b) displays such data together with the $(Q \rightarrow 0)$ value calculated for solid $\mathrm{K}$ at room temperature [14] and shows that liquid data come close to the value for the crystal at long wavelengths. This result is further substantiated by comparing $\omega_{l}$ or $c_{\infty}$ with experimental single-crystal dispersions for longitudinal phonons measured along the [00 $\zeta$ ] direction at $9 \mathrm{~K}, 299 \mathrm{~K}$ [12], as well as to the values extrapolated to $343 \mathrm{~K}$. Figure 3(b) illustrates how the crystal branches approach $c_{\infty} Q$ from below as expected on hydrodynamic grounds. This is understood since phonon dispersions for cubic metals along main symmetry directions are governed by the $V^{\prime}(r), V^{\prime \prime}(r)$ derivatives of $V(r)$ taken at nearest-neighbor distances and the lattice constant, and none of these shows abrupt changes upon melting the hot crystal. 


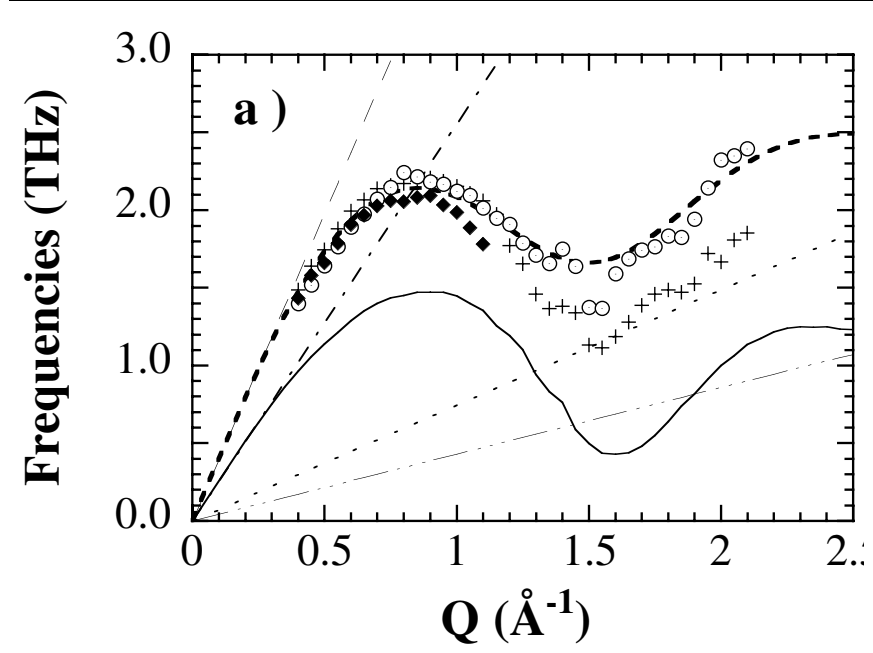

which compare with reported values for liquids of $\approx$ $6500 \mathrm{~ms}^{-1}, 3025 \mathrm{~ms}^{-1}$, and $1061 \mathrm{~ms}^{-1}$, respectively.

The picture drawn from the present data portrays vibratory, short-lived motions in simple liquids explored at $\mathrm{THz}$ frequencies as a remnant of those of their parent crystals at temperatures close to melting. In contrast, liquid dynamics once a configuration becomes unstable exhibits rather more intricacies. While the microscopics of single-particle diffusion are now adequately understood, the dynamic correlations between the tagged particle and its neighbors which take place over many picoseconds still need to be clarified in full.

This work is supported by Grant No. PB98-0673-c02-01 (Spain).

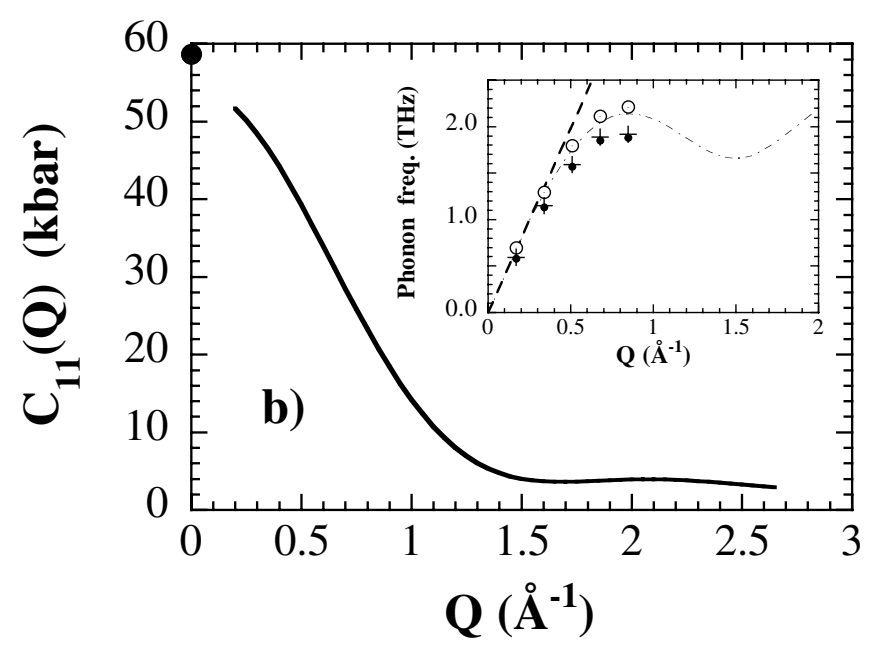

FIG. 3. (a) Estimates for $\omega_{0}$ (solid line). The dash-dotted line shows hydrodynamic dispersion $\Omega_{\text {hyd }}=c_{s} Q$ with $c_{\mathrm{T}}=$ $2.55 \mathrm{THz} \AA$ and the dashes stand for $c_{\infty} Q$ with $c_{\infty}=3.96$ $\mathrm{THz} \AA$. Thick dots are $\omega_{l}$ as calculated from $R_{0}$ and $\omega_{E}$. Circles with a dot show $\omega_{l}$ when left as a free parameter. Filled symbols give $\omega_{m}$ and crosses $\omega_{m l}$ (see text). The dashdotted and dotted lines depict the exact $Q \rightarrow \infty$ ideal-gas limits $\omega_{0}^{\text {i.g. }}=\sqrt{Q^{2} k_{B} T / M}$ and $\omega_{l}^{\text {i.g. }}=\sqrt{3 Q^{2} k_{B} T / M}$ for the two frequency moments. (b) Wave vector dependence of $C_{11}(Q)$ elastic moduli. The solid line shows the data for molten $\mathrm{K}$ as derived from $\omega_{l}$. The thick filled symbol at $Q=0$ shows the value calculated from elastic moduli of the room temperature crystal. The inset compares $c_{\infty}$ (dashes) and $\omega_{l}$ (solid line) with the experimental frequencies for the $\Delta_{5}[00 \zeta]$ dispersion [12] for $9 \mathrm{~K}$ (open circles), $299 \mathrm{~K}$ (crosses), and an extrapolation to $343 \mathrm{~K}$ (solid dots).

Rather than being restricted to $\mathrm{K}$, the interpretation of the high-frequency sound velocity just described can easily be extended to the other alkali metals for which elasticconstant data are available ( $\mathrm{Li}, \mathrm{Na}$, and $\mathrm{Cs}$ [14]). The calculated values for the hot crystals yield phase velocities of $6692 \mathrm{~ms}^{-1}(\mathrm{Li}), 3545 \mathrm{~ms}^{-1}(\mathrm{Na})$, and $1363 \mathrm{~ms}^{-1}(\mathrm{Cs})$,

*Deceased.

${ }^{\dagger}$ Deceased.

[1] N.H. March, Liquid Metals (Cambridge University Press, Cambridge, U.K., 1990).

[2] T. M. Wu and S.F. Tsay, Phys. Rev. B 58, 27 (1998); R. Vallauri and F.J. Bermejo, Phys. Rev. E 51, 2654 (1995).

[3] H. Sinn et al., Phys. Rev. Lett. 78, 1715 (1997); T. Scopigno et al., Phys. Rev. Lett. 85, 4076 (2000); W. Moontfrooij et al., Phys. Rev. A 33, 1405 (1986); W.C. Pilgrim et al., J. Non-Cryst. Solids 250-252, 96 (1999); L. E. Bove et al., Phys. Rev. Lett. 85, 5352 (2000); J. R. D. Copley and J. M. Rowe, Phys. Rev. Lett. 32, 49 (1974); T. Bodensteiner et al., Phys. Rev. A 45, 5709 (1992).

[4] S.W. Lovesey, Theory of Neutron Scattering from Condensed Matter (Oxford Science Publications, Oxford, U.K., 1986), Chap. 5.

[5] K. Miyazaki and I.M. de Schepper, Phys. Rev. Lett. 87, 214502 (2001).

[6] A. G. Novikov et al., Physica (Amsterdam) 228B, 312 (1996); 236B-239B, 359 (1997); J. Phys. Condens. Matter 8, 3525 (1996).

[7] G. Wahnström and L. Sjögren, J. Phys. C 15, 401 (1982).

[8] T. Iida and R. I. L. Guthrie, The Physical Properties of Liquid Metals (Oxford Science Publications, Oxford, U.K., 1993).

[9] E. G. D. Cohen, P. Westerhuijs, and I. M. de Schepper, Phys. Rev. Lett. 59, 2872 (1987).

[10] S. K. Lai, W. Li, and M. P. Tosi, Phys. Rev. A 42, 7289 (1990).

[11] W. van der Lugt, in Handbook of Thermodynamic and Transport Properties of Alkali-Metals, edited by R.W. Ohse (Blackwells, Oxford, 1985).

[12] W. J. L. Buyers and R. A. Cowley, Phys. Rev. 180, 755 (1969); H. R. Glyde, J. P. Hansen, and M. L. Klein, Phys. Rev. B 16, 3476 (1977).

[13] A. B. Bhatia, Ultrasonic Absorption (Dover Publications, Oxford, U.K. 1967), p. 236.

[14] M. M. Beg and M. Nielsen, Phys. Rev. B 14, 4266 (1976); A. D. B. Woods et al., Phys. Rev. 128, 1112 (1962); W.B. Daniels, Phys. Rev. 119, 1246 (1960); N. Nücker and U. Buchenau, Phys. Rev. B 31, 5479 (1985). 